ARTICLE

DOI: $10.1038 / s 41467-017-01400-y$

\title{
Genomic structural variation-mediated allelic suppression causes hybrid male sterility in rice
}

Rongxin Shen ${ }^{1,2,3}$, Lan Wang ${ }^{2,3}$, Xupeng Liư ${ }^{1,2,3}$, Jiang Wu ${ }^{1,2,3}$, Weiwei Jin ${ }^{4}$, Xiucai Zhao ${ }^{1,2,3}$, Xianrong Xie ${ }^{1,2,3}$, Qinlong Zhu ${ }^{1,2,3}$, Huiwu Tang ${ }^{1,2,3}$, Qing Li, ${ }^{1,2,3}$, Letian Chen ${ }^{1,2,3}$ \& Yao-Guang Liu ${ }^{1,2,3}$

Hybrids between divergent populations commonly show hybrid sterility; this reproductive barrier hinders hybrid breeding of the japonica and indica rice (Oryza sativa L.) subspecies. Here we show that structural changes and copy number variation at the Sc locus confer japonica-indica hybrid male sterility. The japonica allele, Sc-j, contains a pollen-essential gene encoding a DUF1618-domain protein; the indica allele, Sc-i, contains two or three tandem-duplicated 28-kb segments, each carrying an Sc-j-homolog with a distinct promoter. In Sc-j/Sc-i hybrids, the high-expression of Sc-i in sporophytic cells causes suppression of Sc-j expression in pollen and selective abortion of $S c-j-$ pollen, leading to transmission ratio distortion. Knocking out one or two of the three Sc-i copies by CRISPR/Cas9 rescues Sc-j expression and male fertility. Our results reveal the gene dosage-dependent allelic suppression as a mechanism of hybrid incompatibility, and provide an effective approach to overcome the reproductive barrier for hybrid breeding.

\footnotetext{
${ }^{1}$ State Key Laboratory for Conservation and Utilization of Subtropical Agro-Bioresources, Guangzhou 510642, China. ${ }^{2}$ Key Laboratory of Plant Functional Genomics and Biotechnology of Guangdong Provincial Higher Education Institutions, Guangzhou 510642, China. ${ }^{3}$ College of Life Sciences, South China Agricultural University, Guangzhou 510642, China. ${ }^{4}$ National Maize Improvement Center of China, Key Laboratory of Crop Genetic Improvement and Genome of Ministry of Agriculture, Beijing Key Laboratory of Crop Genetic Improvement, China Agricultural University, Beijing 100193, China.

Correspondence and requests for materials should be addressed to Y.-G.L. (email: ygliu@scau.edu.cn)
} 
ybrid sterility (HS, including male and female sterilities) reflects the genetic incompatibility between evolutionarily divergent populations in the same or different species. This major form of postzygotic reproductive isolation restricts gene flow and maintains the separation of species (subspecies) during speciation ${ }^{1-3}$. The classical Bateson-Dobzhansky-Muller (BDM) model proposes that the hybrid incompatibility results from deleterious genetic interactions between divergent alleles ${ }^{4-6}$. Asian cultivated rice (Oryza sativa L.) was domesticated from the wild rice (Oryza rufipogon Griff.) and differentiated into two subspecies, japonica and indica ${ }^{7}$. Rice HS, as first reported in 1928, occurs extensively in the inter-subspecific (japonica-indica) hybrids, and is one of the major factors determining the genetic differentiation of these two subspecies ${ }^{8}$.

a

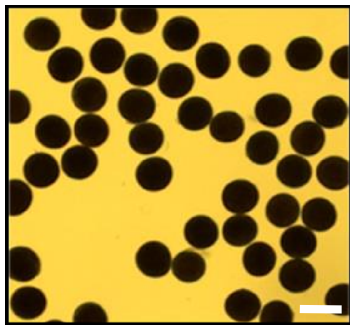

T65 (Sc-j/Sc-j, FF)

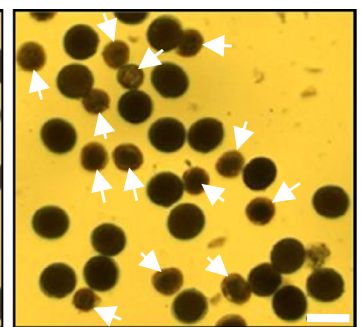

$\mathrm{F}_{1}(\mathrm{Sc}-\mathrm{j} / \mathrm{Sc}-\mathrm{i}, \mathrm{SS})$

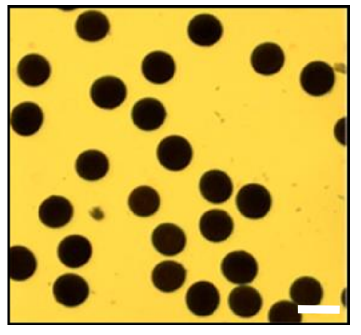

E5 (Sc-i/Sc-i, FF)

$F_{2}, S c-j / S c-j: S c-j / S c-i: S c-i / S c-i$ $=3: 377: 368\left(\chi^{2}=356.3\right)$

b

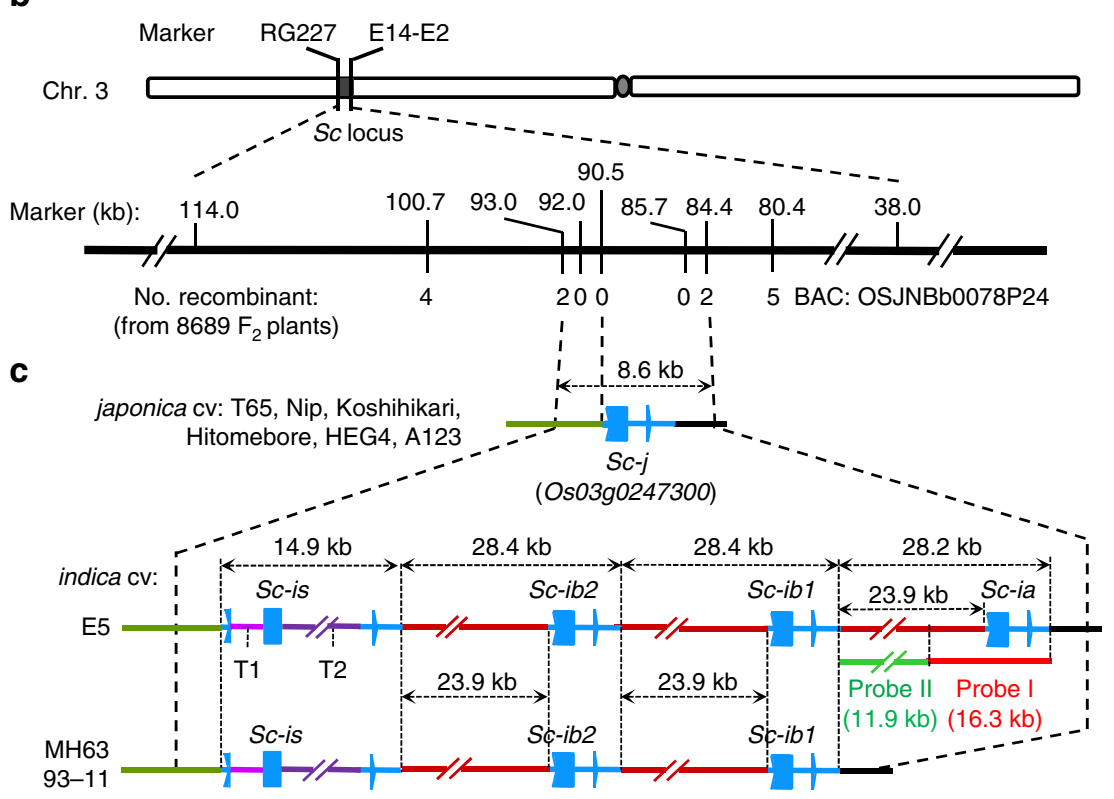

d
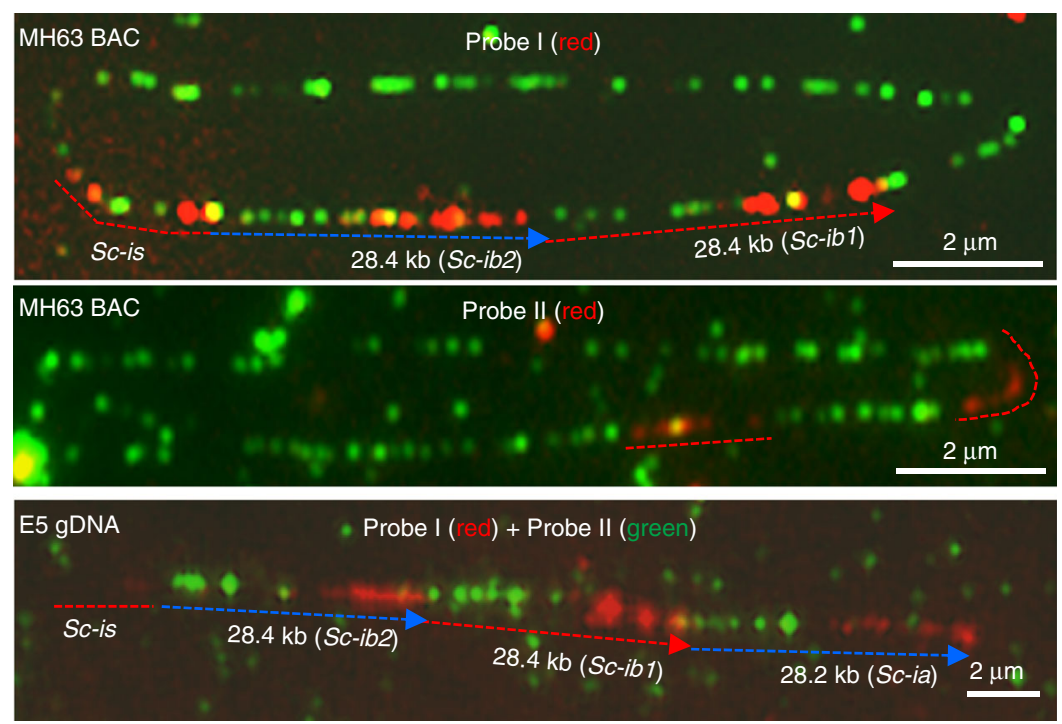
The development and application of hybrid rice technology has contributed greatly to increase rice yield ${ }^{9-11}$. In the past decades, hybrid rice, which boosted grain yield by about $20 \%$ compared to inbred varieties, has been planted ca. 17 million hectares annually in China alone (accounting for about $60 \%$ of its total rice planting area $)^{10}$. However, current hybrid rice varieties were bred mainly from crosses between lines from the same subspecies, mostly indica lines, and hybrid yields have reached a plateau due to the narrow genetic diversity of the parental lines, which results in lower degrees of hybrid vigor (heterosis). By contrast, the hybrids between japonica and indica cultivars have much stronger heterosis, thus holding great promise for further increasing yield potential $^{12}$.

In japonica-indica hybrids, the accumulated genetic effects of multiple HS loci cause very low pollen and spikelet fertilities, which prevents seed formation. Therefore, HS is a major barrier preventing the utilization of the stronger japonica-indica heterosis for achieving higher grain yield, and overcoming HS remains a major challenge in high-yield rice breeding. Genetic studies have identified approximately 50 loci involved in HS in rice $^{3}$, but the allelic relationships of some loci remain unclear. Two general models have been proposed to explain genetic HS in rice: the one-locus sporo-gametophytic interaction model and the duplicate gametic-lethal model ${ }^{13-15}$. The former model proposes that genetic interaction between divergent alleles of a single locus in the sporophytic cells causes abortion of gametes carrying a specific allele. In the later model, epistatic interactions between divergent alleles of two loci cause HS.

So far, the HS-causal genes have been cloned from five loci ( $\mathrm{S} a$, S5, HSA1, S7, and the locus pair DPL1/DPL2) in japonica-indica hybrids $^{16-21}$, from a locus pair $(S 27 / S 28)$ in Asian rice-wild rice (Oryza glumaepatula) hybrids ${ }^{22}$, and from a locus (S1) in hybrids of Asian rice and African rice ${ }^{23}$. Sa confers hybrid male sterility (HMS), and was identified as the first HS complex locus consisting of two adjacent genes $\mathrm{SaF}$ and $\mathrm{SaM}$, which encode an Fbox protein and a small ubiquitin-like modifier E3 ligase, respectively. In $\mathrm{Sa}$-heterozygotes, detrimental interactions among proteins from three alleles (indica $S a F$ and $S a M$, and japonica $\mathrm{SaM}$ ) cause selective abortion of pollen grains carrying the japonica SaM allele, thus establishing the two-gene/three component interaction model at the molecular level ${ }^{16}$. S5 also is a complex locus containing three tightly linked genes, ORF3, ORF4, and ORF5. During female sporogenesis, co-action of the killer/ partner genes ORF4 and ORF5 of the indica allele causes endoplasmic reticulum stress and abortion of embryo sacs that contain the non-functional protector gene ORF3 in the japonica allele; the functional ORF3 of the indica allele rescues these effects ${ }^{18}$. Similarly, the HSA1 locus contains two closely linked genes, $H S A 1 a$ and HSA $1 b$; epistatic interactions among the japonica and indica alleles of the genes in japonica-indica hybrids cause female gamete abortion ${ }^{19}$. At the $S 7$ locus, a gene encoding a tetratricopeptide repeat domain-containing protein confers the Aus-japonica-indica hybrid female sterility ${ }^{20}$. The locus pairs
DPL1/DPL2 and S27/S28 were generated from duplication of the pollen-essential genes on different chromosomes, followed by reciprocal loss of one of the duplicated genes in the divergent species; chromosomal recombination in male meiosis of the hybrids carrying DPL1/DPL2 or S27/S28 produces 25\% sterile pollen grains that lack both of the functional loci ${ }^{21,22}$. At the $S 1$ locus, a gene $O g T P R 1$ encoding a protein containing two trypsinlike peptidase domains and a ribosome biogenesis regulatory domain is required for the interspecific hybrid male and female sterilities $^{23}$. Even though much has been learned about the genetic basis of HS in rice, we still lack a complete picture of its complexity and efficient methods to overcome HS for hybrid rice breeding.

Natural hybrid-compatible (neutral) alleles, which do not cause HS in hybrids, have been found for some HS loci (such as S5- $n$ at $S 5$ and $S a-n$ at $S a$ ) in rice ${ }^{3,14-17}$. However, previous efforts using neutral alleles to develop the inter-subspecific hybrids have had only limited success. In addition, breeding of hybrid widecompatible lines with natural neutral alleles by successive backcrossing is time consuming, and often not feasible because no neutral alleles have been detected for many HS loci.

Genomic variations are the basis for genetic diversity, genome evolution, and natural selection in organisms. Genomic structural variations, including segmental insertion, deletion, inversion, translocation, duplication, and copy number variation (CNV), have significant genetic effects on various biological processes, agronomic traits, and human diseases ${ }^{24,25}$. For example, genomic CNVs increase nematode resistance in soybean ${ }^{26}$ and contribute to grain-size diversity in rice ${ }^{27}$. However, how genomic structural variations generate speciation genes that contribute to reproductive isolation and regulate allele-specific gene expression and function remains largely unclear.

Sc is a major locus conferring HMS in japonica-indica hybrids and was identified using a cross between the japonica cultivar Taichung 65 (T65, carrying a japonica allele, $S c-j$ ) and its nearisogenic line E5 (which has the T65 nuclear background but contains an indica allele, $S c-i$ introgressed from the indica cultivar Peh-ku $)^{13,28}$. We previously mapped $S c$ to a region of chromosome 3 (ref. ${ }^{29}$ ). Here we report the isolation and characterization of the gene for $S c$, and reveal that genomic structural changes and $\mathrm{CNV}$ at $\mathrm{Sc}$ result in allelic suppression that causes the hybrid incompatibility. Based on these findings, we also provide an effective approach that uses genome editing to break down the reproductive barrier for hybrid breeding.

\section{Results}

Identification of a DUF1618 protein gene at the $S c$ locus. The $F_{1}$ hybrid $(S c-j / S c-i)$ of the T65/E5 cross is partially (semi-) sterile due to the abortion of pollen grains carrying $S c-j$, resulting in a severe, male-dependent transmission ratio distortion (mTRD) of the $S c$ alleles (Fig. 1a). Therefore, the $S c-j$ and $S c-i$ alleles are genetically incompatible in the inter-subspecific hybrids. So far,

Fig. 1 Cloning of Sc reveals variations in genomic structure and copy number. a Pollen phenotypes of T65, E5, and their $F_{1}$. Arrows indicate smaller, sterile pollen grains (scale bars, $50 \mu \mathrm{m}$ ). The self-fertilized $F_{2}$ family shows a severely distorted segregation of the alleles $(P<0.001$ for the chi-square test). $\mathbf{b}$ Fine-mapping of Sc using $F_{2}$ plants of the T65/E5 cross. The markers indicate the positions (kb) in the BAC of the japonica cultivar (cv) Nipponbare (Nip). c Genomic configurations of the Sc alleles in japonica lines (from GenBank except for T65) and indica lines (determined in this study). The Sc-i allele variants have three or two tandemly duplicated segments, each containing the gene homologous to Os03g0247300. T1 (2016 bp) and T2 (9087 bp) are transposon insertions (a DDE-type and a Rim2/Hipa-type, respectively). The Sc-i paralogous genes have recombinant promoter/upstream sequences different from that of Sc-j. d Fiber-FISH images of the MH63 BAC ( $180 \mathrm{~kb}$ ) and the E5 chromatin (genomic) DNA (gDNA). For the BAC fiber-FISH, Probes I and II were labeled for red fluorescence, and the whole BAC DNA was labeled for green fluorescence. The labeled Probe I (or Probe II) was mixed with the labeled BAC for the hybridization. For fiber-FISH of E5 gDNA, Probes I and II were labeled for red and green fluorescence, respectively, and mixed for hybridization. Scale bars, $2 \mu \mathrm{m}$. Note that the BAC and chromatin DNA fibers for the same segments had different extension levels, similar to the previous observations $^{34}$. FF full fertility, SS semi-sterility 


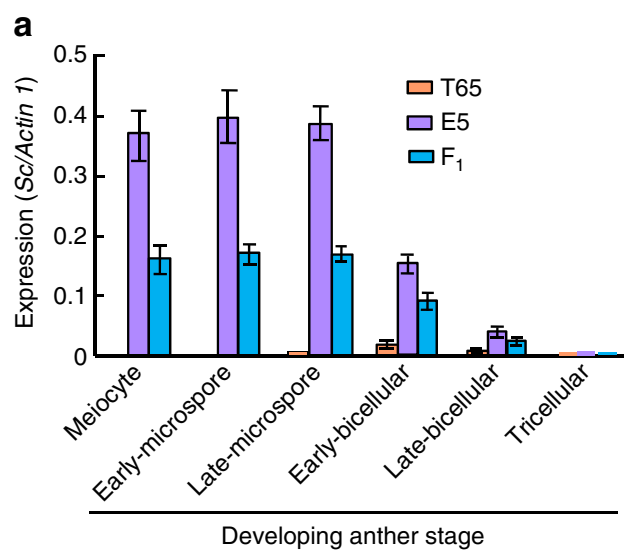

b

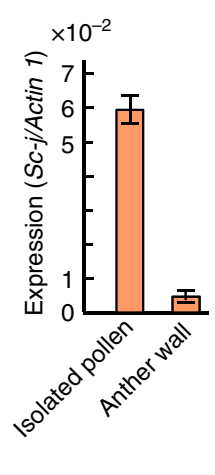

C

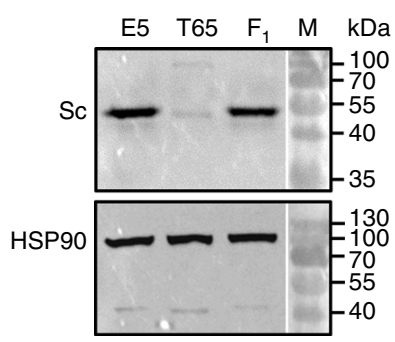

Fig. 2 Expression of the Sc alleles. a Expression of Sc-i and Sc-j in the developing anthers of E5, T65, and their $F_{1}$ by qRT-PCR using a common primer pair (P3/P8, Supplementary Fig. 1) for all the alleles/Sc-i paralogs (except for Sc-is). The values (means of three biological replicates with s.e.m.) were

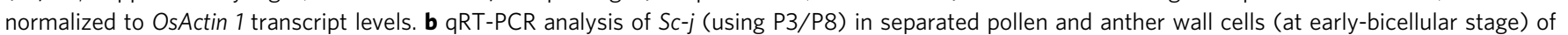

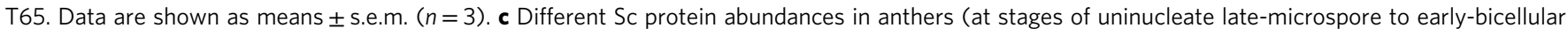
pollen) of the parents and the $F_{1}$, detected with anti-Sc antibodies

no neutral allele at the $S c$ locus has been identified in rice germplasm. By fine-mapping, using the mapping population derived from the T65/E5 cross, we delimited $S c$ to an $8.6-\mathrm{kb}$ region (Fig. 1b). In japonica cultivars, this allele contains an annotated gene Os03g0247300, here named Sc-j (Fig. 1c). Sc-j encodes a DUF1618 domain-containing protein (446 amino acids, aa), belonging to a monocot-specific gene family ${ }^{30}$.

Complex genomic structural variations in the $S c$ - $i$ alleles. Analysis of the genome sequence at the $S c$ locus in the indica cultivar 93-11 (GenBank accession numbers: AAAA02008136.1, AAAA02008130.1, AAAA02008132.1, AAAA02008135.1) suggested complex structural variations at $S c$, but the available sequence in this region was largely incomplete. We therefore sequenced a $S c$ - $i$-containing bacterial artificial chromosome (BAC) of the indica cultivar MH63, and PCR-amplified and sequenced the genomic and cDNA sequences of the $S c-i$ region in 93-11 and E5. The results showed that the complete genomic sequence of the $S c-i$ region in E5 contains four copies of the Os03g0247300 sequence (Fig. 1c and Supplementary Figs. 1, 2). One copy has a similar promoter sequence to $S c-j$ but is a pseudogene (named $S c$-is) due to a 2-bp deletion and two transposon insertions (a DDE-type ${ }^{31}$ and a Rim2/Hipa-type ${ }^{32}$ ) in this gene (Supplementary Fig. 2). The other three copies, named $S c-i a, S c-i b 1$, and $S c-i b 2$, each contain the complete Os03g0247300-homologous sequence, and are located in three tandemly duplicated segments (28.2 or $28.4 \mathrm{~kb}$ in length) (Fig. 1c and Supplementary Figs. 1, 2). MH63 and 93-11 also have $S c$-is and two duplications of the segment $(28.4 \mathrm{~kb})$ with $S c-i b 1$ and $S c$ $i b 2$, but lack the $S c$-ia-containing segment (Fig. 1c). Notably, the 23.9-kb upstream/promoter sequence of $S c-i a, S c-i b 1$, and $S c-i b 2$ differ completely from that of $S c-j$ (Fig. 1c and Supplementary Figs. 1, 2). The coding regions of $S c-j$ and these paralogous $S c-i$ genes include nineteen single-nucleotide polymorphisms (SNPs) and three 3-bp insertion/deletions, which result in several aa substitutions, but the coding sequences of $S c-i b 1$ and $S c-i b 2$ are identical (Supplementary Figs. 3 and 4). The $S c-i$ allele variants probably arose from genomic rearrangement between the ancestral DUF1618 gene and the 23.9-kb genomic sequence to produce a new recombinant gene, followed by inactivation of $\mathrm{Sc}$ is and CNV of the $\sim 28-\mathrm{kb}$ segment.

Using PCR assays, we genotyped 14 additional japonica cultivars and 21 indica cultivars, and found that all tested japonica cultivars have the $S c-j$ allele and all indica cultivars have the $S c$ - $i$ allele with at least two duplications of the segment (Supplementary Fig. 5a-c). We further genotyped this locus in $O$. rufipogon and found that, of the nine accessions analyzed, five have the $S c$-i-type structure (including $S c$-is and at least two duplications of the $\sim 28$-kb segment) and four have the $S c-j$-type structure (Supplementary Fig. 5d). This suggests that the divergence of $S c$ occurred before the domestication of $O$. sativa from $O$. rufipogon and the differentiation of the japonica and indica subspecies.

Since the PCR assay could not determine the actual copy number of the $\sim 28-\mathrm{kb}$ segment at the $S c-i$ allele variants, we further carried out DNA fiber-fluorescence in situ hybridization (fiber-FISH) $)^{33,34}$ to examine the $S c-i$ structure of several indica cultivars. We found that five cultivars (MH63, 93-11, HHZ, Dular, and 02428) contain two duplicates (not including Sc-is) of the segment, whereas three other cultivars (E5, ZS97B, and GLA4) contain three such duplicates (Fig. 1d and Supplementary Fig. 6), consistent with the sequencing results of E5, MH63, and 93-11. These results indicate that the genomic structural change and the segment $\mathrm{CNV}$ at $\mathrm{Sc}$ is a common feature in indica cultivars.

Different expression patterns of the $\boldsymbol{S c}$ - $\boldsymbol{j}$ and $\boldsymbol{S c} \boldsymbol{c} \boldsymbol{i}$ alleles. We speculated that the structural variations might alter the expression pattern and/or level of the diverged $S c$ alleles; therefore, we examined their expression by semi-quantitative and quantitative reverse transcription PCR (qRT-PCR). $S c-j$ in T65 was expressed specifically in anthers, peaking but having a low level at the earlybicellular pollen stage (Fig. $2 \mathrm{a}$ and Supplementary Fig. 7a), which is consistent with the microarray-based transcriptome data from the japonica cultivar Nipponbare (Nip, with $S c-j$ ) (Supplementary Fig. 7b). The repeated $S c-i$ paralogs in E5, however, were expressed broadly in vegetative organs and anthers of different stages, and the total expression level of all paralogs was much higher than that of $S_{c-j}$ (Fig. 2a and Supplementary Fig. 7a). In the early-bicellular stage anthers, the $S c$ - $j$ expression level $(0.020$, expressed as the ratio to OsActin 1 expression) in T65 was only $13 \%$ that of $S c-i(0.151)$ in E5 (Fig. 2a).

To verify if $S c-j$ is expressed in pollen, we carried out mRNA in situ hybridization to $S c-j$ in anthers of T65, but failed to obtain a clear signal, probably due to its very low-level expression. Alternatively, we separated pollen grains from the anther wall (epidermis) cells (at the early-bicellular stage) of T65, The results showed that $S c-j$ was expressed mainly in pollen and showed 
a

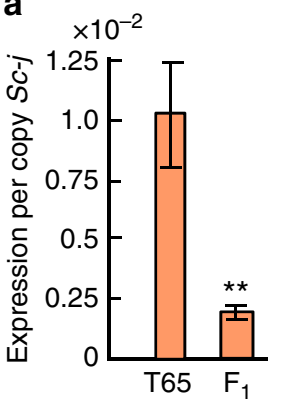

(Anthers) $\times 10^{-2}$

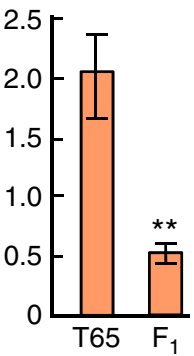

(Isolated pollen) b

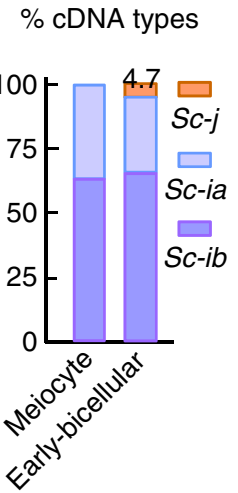

C

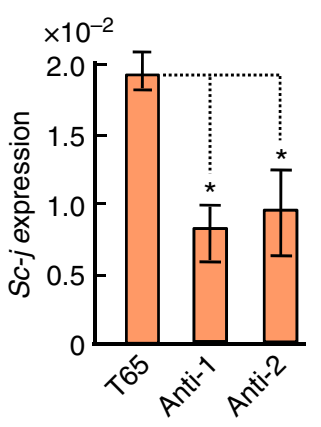

d
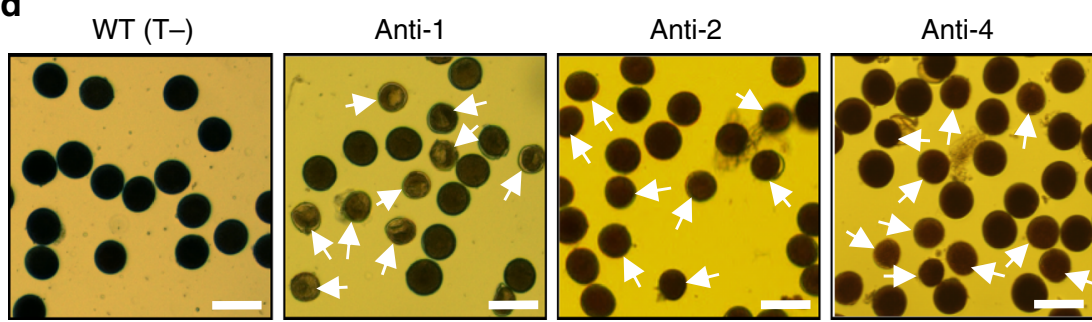

Fig. 3 Functional analysis of Sc-j. a qRT-PCR analysis of relative Sc-j expression levels (using the Sc-j-specific primers P1/P2) in the anthers and isolated pollen (at early-bicellular stage) of the $F_{1}(T 65 / E 5)$ and T65, calculated as the ratios of the transcript per copy Sc-j to the transcript of OsActin 1 (two copies), because there is only one Sc-j copy in the $F_{1}$. The data are shown as means \pm s.e.m. $(n=3)$, and * and ** indicate significance at $P<0.05$ and $P<0.01$, respectively, by two-tailed Student's $t$ test (the same for Fig. 3c). b Percentages of cDNA clones from Sc-j, Sc-ia, and Sc-ib (Sc-ib1/ib2) of the $F_{1}$ anthers of two stages indicated. $\mathbf{c}$ Expression of $S c-j$ in the early-bicellular stage anthers of two $T_{1}$ (T65 background) plants carrying the hemizygous antisense-transgene of Sc-j. The detected transcript levels in Anti- 1 and Anti-2 could be mainly from the pollen grains ( 50\%) without the antisensetransgene. d Semi-sterility of three $T_{1}$ plants carrying the hemizygous antisense-transgene. WT (T-), a $T_{1}$ segregant without the antisense-transgene. Scale bars, $50 \mu \mathrm{m}$

much less expression in the anther wall (Fig. 2b), consistent with its proposed function in pollen development (see below). The differential expression of the $S c-j$ and $S c-i$ alleles was also reflected by their different protein abundances (Fig. $2 c$ and Supplementary Fig. 8). We speculated that the high-level and broad expression patterns of $S c-i$ could result from the repeated gene copies and the distinct promoter sequence.

The expression of $\boldsymbol{S c}-\boldsymbol{j}$ is largely suppressed in the hybrid. To explore the possible causal relationship between the differential expression of the $S c$ alleles and HMS in the hybrid, we examined $S c-j$ expression levels in the hybrid and T65 using $S c$-j-specific primers. In the early-bicellular stage anthers, the expression level of $S c-j$ per copy in the hybrid was about $1 / 5$ of that in T65 (Fig. 3a). Similar results were obtained in isolated early-bicellular stage pollen grains (Fig. 3a), suggesting that $S c-j$ expression is largely repressed in the hybrid pollen grains. We further analyzed the relative transcript abundances of $S c-j$ and the $S c-i$ paralogs in the hybrid by genotyping cDNA clones based on the SNPs in $S c-j$ and the $S c-i$ paralogs (Supplementary Fig. 3). In anthers of the meiocyte (microspore mother cell and meiosis I) and uninucleate microspore stages, no or very few $S c$-j-type cDNA clones were detected; in the early-bicellular stage anthers, $S c-j$ type cDNAs accounted for only $4.7 \%$ of the total clones (Fig. $3 \mathrm{~b}$ and Supplementary Table 1), much lower than the expression level of $S c-j$ in T65 (13\%) compared to E5 (Fig. 2a). Together, these results suggest that normal pollen development requires a threshold level of $S c-j$ expression in the early-bicellular pollen, and that the suppression of expression $S c-j$ in the hybrid, a phenomenon that we term "allelic suppression", might be the cause of $S c$-j-pollen abortion.
Previous cytological observation of sectioning pollen of the T65/E5 hybrid showed that at the bicellular stage, the cell wall (callose) of the mitosis I-generated generative cell (nucleus) within $S c$-j-pollen failed to degenerate at the proper time and the generative nucleus could not move with the vegetative nucleus as occurs in normal bicellular pollen. This leads to the generative nucleus being unable to complete mitosis $\mathrm{II}^{35}$. Therefore, this allelic suppression of $S c-j$ in early-bicellular pollen may be related to the cytological mechanism of impaired $S c$-j-pollen development.

$S c$ is a gametophytic factor for pollen development. To further test if $S c$ is essential for pollen development, we introduced an $S c$ antisense construct into T65 plants. As expected, the hemizygous transgenic plants with reduced $S c-j$ expression in their anthers showed partial male-sterility, similar to the Sc-mediated HMS (Fig. 3c, d). Furthermore, the transgene segregation ratio in five $\mathrm{T}_{1}$ families (from self-fertilization) fitted the 1:1 ratio expected for a hemizygous gametophytic sterility gene (Supplementary Table 2). Therefore, we concluded that this DUF1618 gene has a primary function as a gametophytic factor, and normal pollen development requires a threshold level of $S c$ expression.

To test whether the abnormal development of $S c$-j-pollen involves changes in the expression of genes known to affect male fertility $^{36,37}$, we analyzed a microarray-based transcriptome data set from anthers of T65 and the T65/E5 $\mathrm{F}_{1}$. Of 14 genes with known functions in microspore/pollen development, four (OsADF, OsGSL5, OsUgp2, and OsCAP1) showed differential microarray hybridization signals (Supplementary Fig. 9a). However, further qRT-PCR analysis did not find significant difference in expression of these four genes in developing anthers between T65 and the $\mathrm{F}_{1}$ (Supplementary Fig. 9b). 
Table 1 Segregation distortion in various crosses using indica parents with different copy numbers of Sc-i

\begin{tabular}{|c|c|c|c|c|c|}
\hline ZS97B/Nip & $S c-j / 3 x S c-i$ & 213 & $7: 118: 88$ & 3.3 & $64.1^{\star \star \star}$ \\
\hline Nip/93-11 & $S c-j / 2 \times S c-i$ & 258 & $25: 145: 88$ & 9.7 & $34.7^{\star \star \star}$ \\
\hline $\mathrm{Nip} / 02428$ & $S c-j / 2 \times S c-i$ & $\begin{array}{l}172 \\
(\mathrm{RIL})\end{array}$ & $\begin{array}{l}12: 95: 65 \\
j j: i i\end{array}$ & 7.0 & $\begin{array}{l}34.6^{\star \star \star} \\
(1: 1)\end{array}$ \\
\hline GLA4/Nip & $S c-j / 3 \times S c-i$ & $106\left(F_{10}\right)$ & $1: 105$ & 0.9 & $102.0^{\star \star \star}$ \\
\hline
\end{tabular}

CNV of $\boldsymbol{S c}-\boldsymbol{i}$ is associated with the degree of HMS. To investigate the relationship between the $S c-i \mathrm{CNV}$ and HMS, we crossed the japonica cultivar Nip with indica cultivars that have three copies (E5 and ZS97B) or two copies (93-11, HHZ, and 02428) of the $\sim 28$-kb segments (Supplementary Fig. 6). The two $\mathrm{F}_{2}$ families with three $S c-i$ copies had more severe $\mathrm{mTRD}$ (with $0.4 \%$ and $3.3 \%$ of $S c-j$ homozygotes) than those of the three $F_{2}$ families with two $S c-i$ copies (with $4.7-9.7 \%$ of $S c-j$ homozygotes) (Table 1). Particularly, the recombinant inbred lines (RILs) derived from crossing Nip with the indica cultivar GLA4 (carrying three $S c-i$ copies) produced only $0.9 \% S c-j$ homozygotes, whereas the frequency was $21.1 \%$ in the RILs derived from crossing Nip with 9311 . The more severe mTRD reflects greater abortion of $S c-j$-pollen in the $S c$-heterozygotes. These results suggest that the severity of HMS in the hybrids is associated with the CNV at Sc-i. Notably, all the indica cultivars analyzed have at least two $S c-i$ copies (Supplementary Figs 5 and 6) and these $S c-i$ allele types produced different degrees of genetic incompatibility to japonica cultivars (Table 1).

Reduction of the $\boldsymbol{S c}$ - $\boldsymbol{i}$ dosage rescues the HMS. Based on the relationship of the $S c-i \mathrm{CNV}$ with the HMS, we tested whether decreasing the number of functional $S c-i$ copies by genome editing could generate artificial neutral alleles. Using our CRISPR/ Cas9 plant genome editing system ${ }^{38}$, we made two binary constructs, one to delete the $\sim 28-\mathrm{kb}$ segment(s) and another to specifically edit a unique target site in $S c-i b 1$ and $S c$-ib2 (but not in $S c$-ia) based on a SNP that forms the protospacer adjacent motif (PAM) required for CRISPR/Cas9 genome editing (Fig. 4a). By transformation of E5 with these constructs, we identified a plant (E5-d1) with one of the segmental duplications deleted (Fig. 4a and Supplementary Fig. 6), and two plants (E5-ed1 and E5-ed2) with nucleotide mutations in $S c-i b 1$ and $S c$-ib2, but with their $S c$-ia intact due to the lack of a PAM (Fig. 4a). We crossed these edited plants with T65 to obtain $F_{1}$ plants $\left(F_{1}-d 1, F_{1}\right.$-ed1, and $F_{1}$-ed2) and confirmed the edited sites by sequencing (Supplementary Fig. 10). The pollen fertility of these hybrids was greatly improved (Fig. $4 \mathrm{~b}$ ) and the $\mathrm{F}_{2}$ families showed less mTRD; two $F_{2}$ families from $F_{1}$-ed1 and $F_{1}$-ed2 had segregation ratios fitting the 1:2:1 ratio (Table 2), thus confirming the restoration of $S c-j$-pollen fertility. Therefore, reduction of the gene dosage by the targeted mutation of two of the three $S c-i$ paralogs can convert the hybrid-incompatible $S c-i$ allele into completely compatible alleles while maintaining the basic function of $S c$ in gametogenesis. We name these edited $S c-i$ alleles $S c-n$, as they produce no significant HMS.

To verify the causal relationship between expression of $S c-i$ and $S c-j$ and male fertility in the edited hybrids, we analyzed the mRNA levels of $S c-i a$, the mutated $S c-i b 1$ and $S c-i b 2$, and $S c-j$ in anthers of the edited $F_{1}$ plants using the intact and edited allele- specific primers (Fig. 4a). The mutated mRNA levels were greatly reduced relative to the $S c$-ia transcript (Fig. $4 c$ ), probably due to the common nonsense-mediated mRNA decay mechanism for abnormal mRNAs ${ }^{39}$. In contrast, the $S c-j$ transcript levels (at the early-bicellular stage) of these edited $F_{1}$ plants significantly increased (Fig. 4d). Thus, the $S c-j$ expression level and the pollen fertility in the $S c$-heterozygotes are negatively associated with the $S c-i$ gene dosage and its expression level.

\section{Discussion}

In this study, we isolated and characterized the gene at the HMS locus Sc. Based on the results, we propose a molecular genetic model for the Sc-HMS system. In the wild rice, the pollenessential $S c$ gene underwent structural changes and CNV, and the divergent $S c$ - $j$-type and $S c$ - $i$-type alleles were inherited into the japonica and indica subspecies, respectively, during domestication. In the japonica-indica hybrids, the $S c-i$ paralogs are highly expressed in the sporophytic cells and the product may interact, directly or indirectly, with the promoter region of $S c-j$ by a yetunknown mechanism to mediate the suppression of $S c-j$ expression. This interaction may involve transcriptional-repressive epigenetic modification(s) of the $S c$ - $j$ promoter region, and the modification state (epiallele) may be retained, through meiosis, in the pollen grains, thus leading to the suppression of $S c-j$ transcription in pollen and the allele-specific male-gamete elimination (Fig. 5). This model of gene dosage-dependent HMS is consistent with the partial, sometimes almost complete, restoration of hybrid male fertility produced by reducing the number of copies in the $S_{c-i} i$ locus in the natural allele variants and the genomeedited alleles of $S c-i$.

The change of the promoter sequence of $S c-i$ via structural variations not only alters its gene expression pattern and levels, producing the putative allelic interaction in the sporophytic cells, but might also allow the $S c-i$ paralogs to escape repression by their own products. Thus, the $S c-i$ allele variants have evolved into selfish speciation genes in this HMS system, and can cause specific abortion of pollen with $S c-j$ but not pollen carrying the $S c-i$ variants in the hybrids. Such a scenario exemplifies the molecular genetic basis of the classical one-locus sporo-gametophytic interaction model for plant $\mathrm{HS}^{13-15}$. The BDM-type genetic incompatibility results from the divergence and interaction of genetic loci during evolution. It would be interesting to know if other intermediate types of $S c$ - $i$-like and/or $S c$-j-like allele variants that are likely hybrid-neutral or hybrid-incompatible exist in wild and cultivated rice populations, for example, the primarily diverged $S c$ - $i$-like allele having a novel, recombinant promoter but without the segmental duplication. Therefore, the Sc-HMS system may be an ideal model for study of BDM-type or a novel type of postzygotic hybrid incompatibility caused by a single locus. Further investigation will be required to analyze the 
a

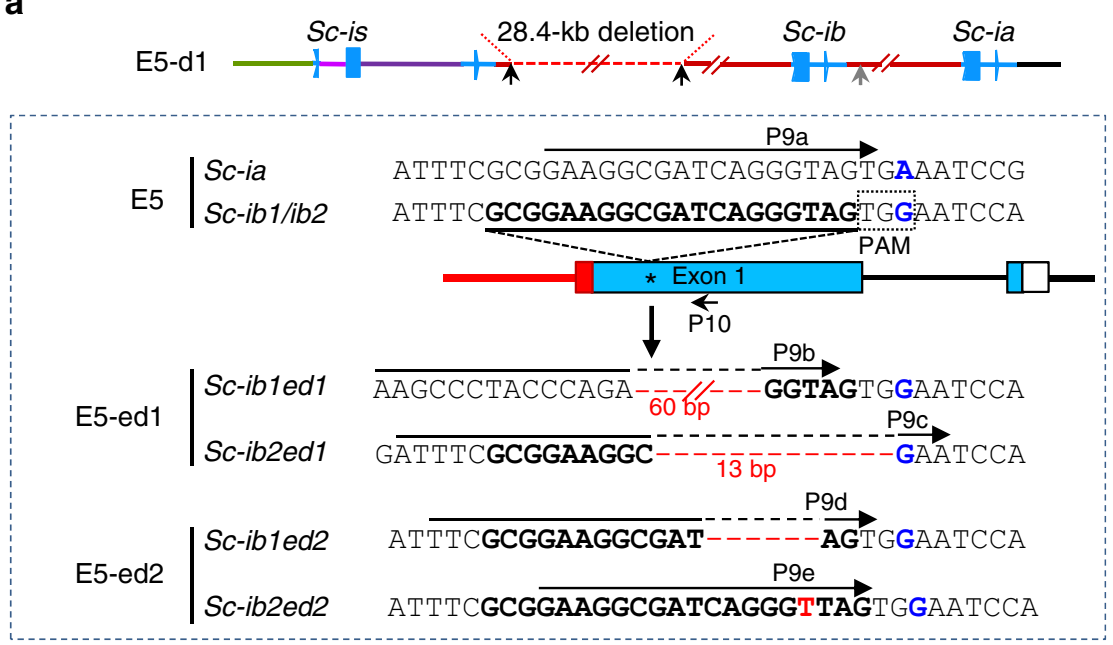

b

Fertile pollen rate (observed pollen grains)

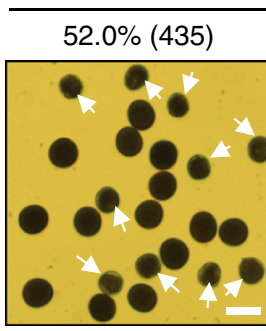

$\mathrm{F}_{1}$
$70.1 \%(365)$

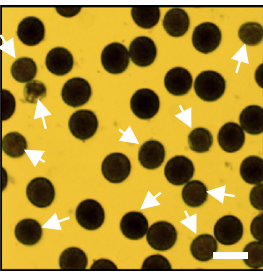

$\mathrm{F}_{1}-\mathrm{d} 1$
$91.8 \%(489)$

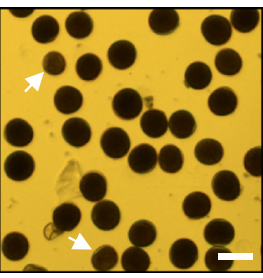

$\mathrm{F}_{1}$-ed1

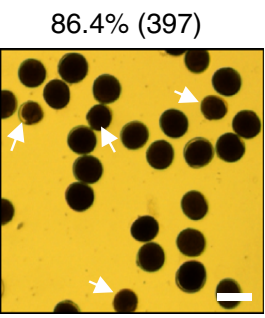

$\mathrm{F}_{1}$-ed2

C

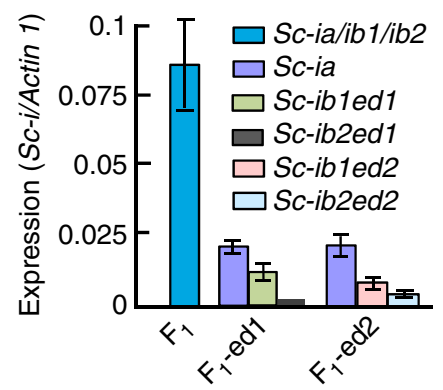

d

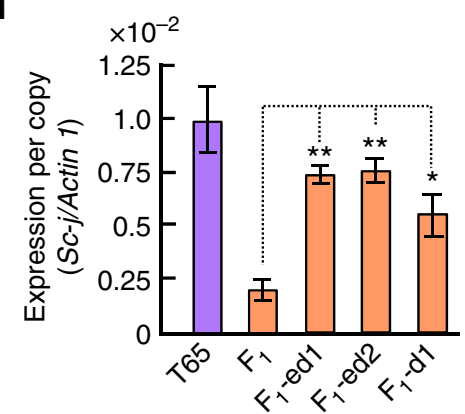

Fig. 4 Genomic editing of the Sc-i paralogs rescues Sc-j expression and male fertility in the hybrids. a Targeted editing of the sites (arrowed) in E5 produced a plant (E5-d1) with deletion of one of the 28-kb segments (Supplementary Fig. 6), and editing another site (underlined) specific to Sc-ib1 and Sc-ib2 of E5 identified two plants (E5-ed1 and E5-ed2) with mutated Sc-ib1 and Sc-ib2 and intact Sc-ia (Supplementary Fig. 10). The SNP (blue) in Sc-ib1 and Sc-ib2 formed the PAM (NGG) required for genome editing. Primers P9a-P9e combined with the Sc-i-specific P10 were used for qRT-PCR of the intact and mutated Sc-i transcripts show in (c). b Improvement of pollen fertility (sterile pollen grains arrowed) in the edited $F_{1}$ plants from crossing E5-d1, E5-ed1, and E5-ed2 with T65. Scale bars, $50 \mu \mathrm{m}$. c Expression levels (in the meiocyte stage anthers) of the Sc-i paralogs in the $F_{1}(T 65 / E 5)$, and the intact Sc-ia and mutated Sc-ib1/Sc-ib2 in the edited F plants. Data are shown as means \pm s.e.m. $(n=3)$. d Expression levels of per copy Sc-j (qRT-PCR using P1/P2) in the early-bicellular stage anthers of the intact and edited $F_{1}$ plants and T65. The data are shown as means \pm s.e.m. $(n=3)$, and * and ** indicate significance at $P<0.05$ and $P<0.01$, respectively, by two-tailed Student's $t$ test

\section{Table 2 Sc genotypic segregation in the edited $F_{2}$ families}

\begin{tabular}{lllll}
$\mathbf{F}_{\mathbf{2}}$ family & No. plant & Segregation jj:ji:ii & \% jj plant & $\chi^{\mathbf{2}}$ (1:2:1) \\
\hline$F_{2}$ & 176 & $1: 93: 82$ & 0.6 & $75.1^{\star \star \star}$ \\
$F_{2}$-d1 & 262 & $32: 141: 89$ & 8.4 & $26.3^{\star \star}$ \\
$F_{2}$-ed1 & 256 & $54: 133: 69$ & 20.5 & 2.1 \\
$F_{2}$-ed2 & 199 & $36: 107: 56$ & 18.1 & 5.15 \\
\hline
\end{tabular}

Note: $j j, i i$, and $j i$ denote homozygous $S c-j$, homozygous $S c-i$ (intact or edited), and the heterozygote, respectively. ${ }^{\star \star}$ and ${ }^{\star * \star}$, significance at $P<0.01$ and $P<0.001$, respectively, for the $\chi^{2}$ test allelic interaction and the molecular mechanism of $S c$, as well as its evolution.

The development of anthers and pollen involves a large number of genes, and many of them are highly conserved between monocot and dicot plant species ${ }^{36,37}$. However, this study revealed that the $S c$ gene is a member of the monocotspecific DUF1618 gene family (121 members in japonica rice). To date, only two genes of this family in rice, $H S A 1 a^{19}$ and $S c$, have been functionally studied. These studies showed that HSA1a and Sc have important roles in reproductive development and participate in hybrid female and male sterilities, respectively. These 


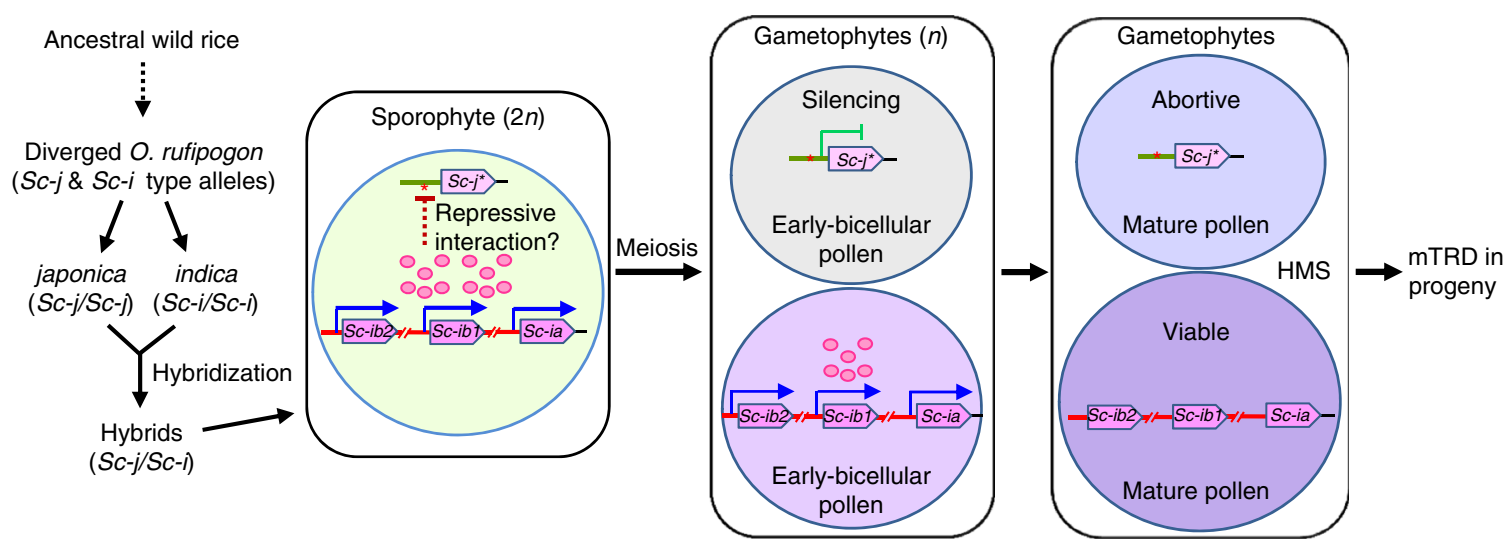

Fig. 5 A proposed model for the molecular genetic mechanism of Sc-mediated HMS. In a japonica-indica hybrid, the Sc-i paralogs are highly expressed in sporophytic cells and the product may interact with the Sc-j promoter region. This might cause transcriptional-repressive epigenetic modification(s) (red asterisk) in the Sc-j promoter region. This putative modification state $\left(S_{c}-j^{\star}\right)$ could be retained, through meiosis, in the male gametophytes, thus causing allelic suppression of Sc-j expression and the consequent Sc-j-specific pollen abortion (HMS) and mTRD. Only two of the four pollen grains from a meiocyte, and one Sc-j copy and one set of Sc-i copies in the bicellular and tricellular pollen grains, are shown

findings demonstrate that rice has evolved genetic networks distinct from those of dicots for male and female gametogenesis.

The findings from studies of a number of cloned HS loci in plants suggest that hybrid incompatibility involves diverse molecular genetic mechanisms ${ }^{40}$; the only common feature among some hybrid incompatibility systems is that they are caused by gene duplication/reciprocal loss-of-function mutations, as shown in the DPL1/DPL2 and $S 27 / 28$ locus pairs ${ }^{21,22}$. In this study we revealed a new type of combinatorial genomic variation involving structural changes and $\mathrm{CNV}$, and demonstrate that this type has an important genetic effect on hybrid incompatibility. Our results show that the $S c-j$ and $S c-i$ alleles are associated with japonica and indica cultivars, suggesting that this hybrid incompatibility might have contributed to the differentiation of the subspecies, although extensive analysis will be required to resolve this issue. The allelic suppression described here suggests a regulatory mode for differential allelic expression, which is characterized by allele-specific $(S c-j)$ repression and is driven by the opposite allele $(S c-i)$ of the same gene. This phenomenon has features distinct from the previously described paramutations ${ }^{41}$ and monoallelic expression forms (parent-of-origin imprinting, random monoallelic expression of autosomal genes, and $\mathrm{X}$-inactivation genes) $)^{42-44}$.

In this study, we used CRISPR/Cas9 genome-editing technology to create neutral alleles of $S c$ by precisely reducing the gene dosage of $S c-i$ but maintaining its basic function in pollen development. By contrast, precise down-regulation of target genes remains difficult to achieve with other methods, such as antisense and RNA interference. The genome editing-based approach can rapidly generate neutral alleles at $S c$ and other HS loci that require loss-of-function mutations to generate neutral alleles, such as $\mathrm{Sa}, \mathrm{S} 5$, and $S 1^{16,17,23,45}$. This may enable breeding of wide-compatible lines to break down the reproductive barrier and overcome HS in the inter-subspecific and inter-specific hybrid breeding in crops, and thus may have significant implications for agriculture.

\section{Methods}

Rice materials. Rice materials for the gene cloning and functional analysis included the japonica line T65 and its near-isogenic line $\mathrm{E} 5^{13,28}$ and their hybrid progeny prepared in this study. The other indica cultivars MH63, ZS97B, HHZ, and GLA4 are Minghui 63, Zhengshan 97B, Huanghuazhan, and Guangluai 4, respectively. The line 02428 with a majority japonica background was derived from a japonica-indica cross ${ }^{46}$. The wild and cultivated rice species used for the haplotype survey and crosses were maintained in our laboratory. The rice plants were planted in experimental fields or in a phytotron (for some seedlings) at the South China Agricultural University in Guangzhou.

Map-based cloning. $F_{2}$ plants of the T65/E5 cross were used for mapping. The pollen phenotypes (male fertile or male sterile) of rice were observed by placing pollen in a $1 \% \mathrm{I}_{2}$-KI (potassium iodide) solution and observing the pollen under a microscope (Leica DMLB, Zeiss Axio Observer D1). Plants homozygous for Sc produced fully fertile pollen ( $>95 \%$ pollen fertile); plants heterozygous for $S c$ produced semi-fertile pollen (ca. $50 \%$ of pollen grains sterile). A number of insertion/deletion markers (Supplementary Table 3) were used for the finemapping. The key recombinant plants (Fig. 1b) were further confirmed by analyzing the phenotypic and genotypic segregation in their $\mathrm{F}_{3}$ progenies. Genetic segregation of the genotypes in various populations was analyzed by $\chi^{2}$ test.

Sequence analysis. The BAC clone (OSIMNBa0008K19) was from an MH63 genomic library ${ }^{47}$. With the incomplete genomic sequences of the indica 93-11 at the $S c$ region (GenBank accession numbers: AAAA02008136.1, AAAA02008130.1, AAAA02008132.1, AAAA02008135.1) as references, a number of primer pairs were prepared and long-length PCR with KOD FX DNA polymerase (TOYOBO, Japan) was used to amplify the $S c-i$ region sequences from the BAC and from gDNAs of E5 and 93-11 in multiple fragments. The PCR products were sub-cloned into a plasmid vector and multiple clones for each fragment were sequenced. The sequences were assembled using SeqMan of the Lasergene package, and analyzed by BLAST (http://www.ncbi.nlm.nih.gov/BLAST/). The DDE-type and Rim2/Hipatype transposon insertions in $S c$-is were analyzed by BLAST (http://www.ncbi.nlm. nih.gov/BLAST/).

DNA fiber-FISH. For BAC fiber-FISH, the BAC plasmid was isolated from $200 \mathrm{ml}$ LB culture using the alkaline lysis method. The BAC DNA was diluted to about $10 \mathrm{ng} / \mu \mathrm{l}$ in AF solution (100\% glycerin: $1 \mathrm{M}$ sodium chloride:double-distilled water, 180:3:17, v/v, pH 7.5) with cut pipet tips. The diluted BAC DNA $(2 \mu \mathrm{L})$ was added to Poly-Prep slides (Sigma-Aldrich, USA), then the slides were slowly covered with coverslips $(22 \times 22 \mathrm{~mm})$ and set vertically to stretch the BAC DNA. The slides were air-dried for $10 \mathrm{~min}$, then carefully uncovered by removing the coverslips. The slides were fixed in fresh Carnoy's solution (ethanol:glacial acetic acid, 3:1, v/v). Probe I ( $16.3 \mathrm{~kb})$ and Probe $2(11.9 \mathrm{~kb})$ DNAs were amplified by PCR in three and two segments, respectively (primer sequences are provided in Supplementary Table 3), and purified on a $1 \%$ agarose gel with a gel-extraction kit. According to the DNA fiber-FISH protocol ${ }^{33}$, the probe sequences (Probe I and Probe II) were labeled by a nick translation reaction with digoxigenin-11-dUTP (Roche, Switzerland), and mixed with the whole BAC DNA labeled with biotin-16-dUTP (Roche, Switzerland) for two separate hybridizations. For hybridization, the probes were firstly added to the slides and covered with the cover slips. Then the slides were denatured at $80^{\circ} \mathrm{C}$ in for $5 \mathrm{~min}$ in an oven. Then the slides were immediately transferred to another oven and incubated at $37^{\circ} \mathrm{C}$ overnight. Immunodetection of the probes were performed by sequentially adding various antibodies to gain amplifying the fluorescence signals. The biotin-labeled probes were detected successively with fluorescein-avidin D (Vector, USA), biotinylated anti-avidin antibody (Vector, USA), and fluorescein-avidin D (Vector, USA). The digoxigeninlabeled probes were firstly detected by anti-digoxigenin-rhodamine (Roche, Switzerland), then by texas red ${ }^{\circledR}$ goat anti-rabbit IgG antibody (Vector, USA). The slides were counterstained with $4^{\prime}$,6-diamidino-2-phenylindole (DAPI) in vectashield mounting medium (Vector, USA). 
For genomic DNA fiber-FISH, nuclei were prepared from young rice seedling leaves, using the plant nuclei isolation method ${ }^{48}$. The seedling leaves were grounded to powder in liquid nitrogen, and the powder was suspended in buffer (10 mM Tris- $\mathrm{HCl}$ (pH 9.5), $10 \mathrm{mM}$ EDTA (pH 8.0), $100 \mathrm{mM} \mathrm{KCl,} 0.5 \mathrm{M}$ sucrose, $4 \mathrm{mM}$ spermidine, $1 \mathrm{mM}$ spermine, $0.1 \%$ mercaptoethanol). After filtering sequentially through 149,74 and $37 \mu \mathrm{m}$ nylon meshes, the nuclei were collected by centrifuging at $1500 \mathrm{~g}$ for $10 \mathrm{~min}$. The genomic DNA fiber-FISH was performed following the protocol ${ }^{34}$. Briefly, the DNA fibers were extended by dragging with a cover slip upon the Poly-L-lysine (Sigma-Aldrich, USA) slides to get long and clean DNA fibers. The slides were hybridized using mixed Probe I labeled with digoxigenin-11-dUTP and Probe II labeled with biotin-16-UTP. The following procedures of hybridization and probes detection were carried as the same as the above description for BAC fiber-FISH. The fiber-FISH images of the BAC and genomic DNAs were captured digitally using a CCD camera (QIMAGING, USA) attached to an Olympus BX61 epifluorescence microscope. Gray-scale images were captured for each color channel and then merged. The final image optimization was performed using Adobe Photoshop CS3 (Adobe, USA).

\section{Immunoblot analysis. A peptide antigen (MVEGNEGEESTKRMKVK) corre-} sponding to amino acid residues 427-443 of Sc-i (430-446 of Sc-j) was synthesized and used for immunizing rabbits to produce the anti-Sc antibodies by Invitrogen (USA) as a customary service.

Detection of Sc-j and Sc-i with the antiserum was carried out as described with some modifications ${ }^{49}$. About $50 \mathrm{mg}$ anthers (at stages of late-microspore to earlybicellular pollen) of T65, E5, and their hybrid $\mathrm{F}_{1}$ were grinded to fine powder in liquid nitrogen and lysed in $500 \mu \mathrm{l} 2 \times$ protein extraction buffer $(100 \mathrm{mM}$ Tris-HCl (pH 7.5), $300 \mathrm{mM} \mathrm{NaCl}, 2 \mathrm{mM}$ EDTA, $10 \%$ glycerol, $0.5 \%$ TritonX-100 and protease inhibitor cocktail from Roche, PMSF from AMRESCO). After centrifugation at $12,000 \mathrm{~g}$ for $15 \mathrm{~min}$, the supernatants were collected and the total proteins were quantified with the 2-D Quant Kit (GE Healthcare, USA). Extracted proteins were mixed with an equal volume of the $2 \times$ loading buffer $(100 \mathrm{mM}$ Tris$\mathrm{HCl}, 5 \mathrm{mM} \mathrm{DTT}, 4 \%$ SDS, $0.01 \%$ bromophenol blue and $30 \%$ glycerol, $\mathrm{pH} 6.8$ ) and boiled for $5 \mathrm{~min}$, then separated by $12 \%$ SDS-PAGE and transferred onto an Immobilon-PPSQP transfer membrane (polyvinylidene fluoride (PVDF) type, Millipore) using a Bio-Rad mini-transfer cell. The membranes were incubated in blocking buffer (5\% milk, $0.1 \%$ Tween-20, $0.1 \%$ Triton X-100, $100 \mathrm{mM}$ Tris- $\mathrm{HCl}$ and $150 \mathrm{mM} \mathrm{NaCl}, \mathrm{pH} 7.5)$ for $2 \mathrm{~h}$ at room temperature, washed twice with $2 \times$ TBST buffer (0.1\% Tween-20, $100 \mathrm{mM}$ Tris- $\mathrm{HCl}$ and $150 \mathrm{mM} \mathrm{NaCl}, \mathrm{pH}$ 7.5). Then the membranes were incubated with anti-Sc polyclonal antibodies (1:2000 dilution) or the anti-Hsp90 monoclonal antibody (Beijing Protein Innovation, China; AbM51099-31-PU) diluted (1:10000) in blocking buffer for about $20 \mathrm{~h}$ at $4{ }^{\circ} \mathrm{C}$. After washing three times with TBST for $5 \mathrm{~min}$ each, the membranes were incubated in the secondary antibodies, HRP-conjugated goat anti-rabbit IgG or HRP-conjugated goat anti-mouse IgG (TransGen Biotech, China), with 1:10000 dilution for $1 \mathrm{~h}$ at room temperature and washed three times with TBST for $5 \mathrm{~min}$ each. The membrane blots were incubated in the ECL substrate (Bio-rad, USA) for $1 \mathrm{~min}$. The photos were captured using Gel Logic 6000 Pro (Kodak, USA) and processed with Adobe Photoshop CS3 (Adobe, USA).

Expression analysis. Separation of pollen grains from anther wall cells was carried out by filtering broken anthers in $2 \%$ sucrose through a nylon mesh $(48 \mu \mathrm{m}$ in diameter), followed by centrifugation at $250 \mathrm{~g}$ for $10 \mathrm{~min}$ to pellet pollen. The separated pollen grains and anther wall cells were collected for RNA preparation. Total RNA was extracted from rice tissues (anthers, isolated pollen, anther wall cells, young panicles, leaves, stems, and roots) of T65, E5, and their hybrid using TRIzol reagent (Invitrogen, USA). The RNA samples were treated with DNase I (Promega, USA). First-strand cDNA was synthesized from $2 \sim 3 \mu \mathrm{g}$ total RNA using Superscript III reverse transcriptase (Invitrogen, USA) with oligo dT or a $S c$ specific primer. The expressions of $S c$ and the pollen-essential marker genes were investigated by qRT-PCR analysis with OsActin 1 (Os03g0718100) for normalization by three biological replicates, and the significance was analyzed by twotailed Student's $t$ test. SYBR Green QPCR mix (Bio-Rad) was used for qRT-PCR. The reactions were carried out using the Bio-rad CFX Connect Real-Time PCR System with the following profile: $95^{\circ} \mathrm{C}$ for $3 \mathrm{~min} ; 42$ cycles of $95^{\circ} \mathrm{C}$ for $10 \mathrm{~s}, 60^{\circ} \mathrm{C}$ for $15 \mathrm{~s}, 72^{\circ} \mathrm{C}$ for $20 \mathrm{~s}$. The expression pattern of $S c-j$ (Os03g0247300) in Nip was from the Rice Expression Profile Database (http://ricexpro.dna.affrc.go.jp/) ${ }^{50}$.

For precise quantification of the ratio of relative $S c-j$ and $S c-i$ copy numbers and transcript levels in the $F_{1}$, the gDNA and cDNA segments that included all the alleles/paralogs in different stage anthers of the $\mathrm{F}_{1}$ plants were amplified using primers $\mathrm{P} 5 / \mathrm{P} 7$. The PCR products were cloned into a plasmid vector, and multiple clones (196-380) for each sample were genotyped. The insertion/deletion marker 3-N1/3-N2 (Supplementary Table 3) was used to differentiate $S c$-j from $S c$-ia/Sc$i b 1 / S c-i b 2$, and the SNP marker HRM1F/HRM1R to differentiate $S c$-ia from $S c-i b 1 /$ Sc-ib2 (Supplementary Fig. 8). SNP genotype was detected by high-resolution melting analysis (LightScanner, USA) using EvaGreen (Biotium, USA) as a fluorescent dye $e^{51}$.

Constructs for rice transformation. A 771-bp fragment covering the 3' coding region of $S c-j$ (from +514 to $+1284 \mathrm{bp}$ ) was amplified and inserted in reverse orientation into an overexpression vector with the maize Ubiquitin promoter to prepare an antisense expression construct. Two genome-targeting constructs for fragmental deletion (the target site: 5'-GGCACAATGAGCGGGCTGGATGG-3', PAM underlined) and Sc-ib1/ib2 mutation (Fig. 4a) were prepared using the CRISPR/Cas9 vector system ${ }^{38}$. These target site sequences were searched against the rice genome sequence with the web-based tool CRISPR-GE/targetDesign (http://skl.scau.edu.cn/ $)^{52}$ to confirm its specificity in the genome. The targetadapter primers are listed in Supplementary Table 3. All constructs were introduced into Agrobacterium tumefaciens strain EHA105 by electroporation and used for rice transformation. The PCR amplicons containing the target sites were directly sequenced and cloned in a plasmid for sequencing. The superimposed chromatograms of the direct sequencing were decoded with CRISPR-GE/DSDe$\operatorname{codeM}^{52,53}$, and the PCR products also were cloned in a plasmid vector and multiple clones were sequenced.

Data availability. The nucleotide sequence data for $S c-j$ of T65 and the $S c-i$ variants of E5 and MH63 have been deposited in GenBank nucleotide database under accession numbers MF370526, KX495643 and KX495644, respectively. All the relevant data are available from the authors upon request.

Received: 26 April 2017 Accepted: 14 September 2017

Published online: 03 November 2017

\section{References}

1. Bomblies, K. Doomed lovers: mechanisms of isolation and incompatibility in plants. Annu. Rev. Plant. Biol. 61, 109-124 (2010).

2. Nosil, P. \& Schluter, D. The genes underlying the process of speciation. Trends Ecol. Evol. 26, 161-167 (2011).

3. Ouyang, Y. \& Zhang, Q. Understanding reproductive isolation based on the rice model. Annu. Rev. Plant. Biol. 64, 111-135 (2013).

4. Bateson, W. in Darwin and modern science (ed. Seward, A. C.) 85-101 (Cambridge Univ. Press, Cambridge, 1909).

5. Dobzhansky, T. Genetics and the origin of species (Columbia University Press, New York, 1937).

6. Muller, H. J. Isolating mechanisms, evolution and temperature. Biol. Symp. 6 , 71-125 (1942)

7. Huang, X. et al. A map of rice genome variation reveals the origin of cultivated rice. Nature 490, 497-501 (2012).

8. Kato, S., Kosaka, H. \& Hara, S. On the affinity of rice varieties as shown by fertility of hybrid plants. Bull. Sci. Fac. Agric. Kyushu Univ. 3, 132-147 (1928).

9. Yuan, L. Hybrid Rice Technology: New Developments and Future Prospects. 1-6 (International Rice Research Institute, Philippines, 1994).

10. Cheng, S., Zhuang, J., Fan, Y., Du, J. \& Cao, L. Progress in research and development on hybrid rice: a super-domesticate in China. Ann. Bot. 100, 959-966 (2007).

11. Yuan, L. Development of hybrid rice to ensure food security. Rice Sci. 21, 1-2 (2014).

12. Qian, Q., Guo, L., Smith, S. \& Li, J. Breeding high-yield superior-quality hybrid super-rice by rational design. Natl Sci. Rev. 3, 283-294 (2016).

13. Oka, $\mathrm{H}$. Analysis of genes controlling $\mathrm{F}_{1}$ sterility in rice by the use of isogenic lines. Genetics 77, 521-534 (1974).

14. Ikehashi, H. \& Araki, H. Genetics of $F_{1}$ sterility in remote crosses of rice. Rice Genet. 119-130 (1986).

15. Sano, Y. The genic nature of gamete eliminator in rice. Genetics. 125, 183-191 (1990).

16. Long, Y. et al. Hybrid male sterility in rice controlled by interaction between divergent alleles of two adjacent genes. Proc Natl Acad. Sci. USA 105 18871-18876 (2008).

17. Chen, J. et al. A triallelic system of $S 5$ is a major regulator of the reproductive barrier and compatibility of japonica-indica hybrids in rice. Proc. Natl Acad. Sci. USA 105, 11436-11441 (2008).

18. Yang, J. et al. A Killer-Protector system regulates both hybrid sterility and segregation distortion in rice. Science 337, 1336-1340 (2012).

19. Kubo, T., Takashi, T., Ashikari, M., Yoshimura, A. \& Kurata, N. Two tightly linked genes at the hsal locus cause both $\mathrm{F}_{1}$ and $\mathrm{F}_{2}$ hybrid sterility in rice. Mol. Plant 9, 221-232 (2016)

20. Yu, Y. et al. Hybrid sterility in rice (Oryza sativa L.) involves the tetratricopeptide repeat domain containing protein. Genetics 203, 1439-1451 (2016).

21. Mizuta, Y., Harushima, Y. \& Kurata, N. Rice pollen hybrid incompatibility caused by reciprocal gene loss of duplicated genes. Proc Natl Acad Sci USA 107, 20417-20422 (2010).

22. Yamagata, Y. et al. Mitochondrial gene in the nuclear genome induces reproductive barrier in rice. Proc Natl Acad Sci USA 107, 1494-1499 (2010). 
23. Xie, Y. et al. Interspecific hybrid sterility in rice is mediated by OgTPR1 at the S1 locus encoding a peptidase-like protein. Mol. Plant 10, 1137-1140 (2017).

24. Weischenfeldt, J., Symmons, O., Spitz, F. \& Korbel, J. O. Phenotypic impact of genomic structural variation: insights from and for human disease. Nat. Rev. Genet. 14, 125-138 (2013).

25. Alkan, C., Coe, B. P. \& Eichle, E. E. Genome structural variation discovery and genotyping. Nat. Rev. Genet. 12, 363-375 (2011).

26. Cook, D. E. et al. Copy number variation of multiple genes at Rhgl mediates nematode resistance in soybean. Science 338, 1206-1209 (2012).

27. Wang, Y. et al. Copy number variation at the GL7 locus contributes to grain size diversity in rice. Nat. Genet. 47, 944-948 (2015).

28. Zhang, G., Lu, Y., Zhang, H., Yang, J. \& Liu, G. Genetic studies on the hybrid sterility in cultivated rice (Oryza sativa L.) IV. Genotypes for $\mathrm{F}_{1}$ pollen sterility. Acta Genet. Sinica 21, 34-41 (1994).

29. Yang, C., Chen, Z., Zhuang, C., Mei, M. \& Liu, Y.-G. Genetic and physical finemapping of the $S c$ locus conferring indica-japonica hybrid sterility in rice (Oryza sativa L.). Chinese Sci. Bull. 49, 1718-1721 (2004).

30. Wang, L., Shen, R., Chen, L. \& Liu, Y.-G. Characterization of a novel DUF1618 gene family in rice. J. Integr. Plant Biol. 56, 151-158 (2014).

31. Marchler-Bauer, A. et al. CDD: NCBI's conserved domain database. Nucleic Acids Res. 43, D222-D226 (2015).

32. Wang, G. D. et al. Genomic characterization of Rim2/Hipa elements reveals a CACTA-like transposon superfamily with unique features in the rice genome. Mol. Genet. Genomics 270, 234-242 (2003).

33. Jackson, S. A. et al. Application of fiber-FISH in physical mapping of Arabidopsis thaliana. Genome 41, 566-572 (1998).

34. Cheng, Z. et al. Functional rice centromeres are marked by a satellite repeat and a centromere-specific retrotransposon. Plant Cell 14, 1691-1704 (2002).

35. Zhang, Z., Lu, Y., Liu, X., Feng, J. \& Zhang, G. Cytological mechanism of pollen abortion resulting from allelic interaction of $\mathrm{F}_{1}$ pollen sterility locus in rice (Oryza sativa L.). Genetica 127, 295-302 (2006).

36. Guo, J. \& Liu, Y.-G. Molecular control of male reproductive development and pollen fertility in rice. J. Integr. Plant Biol. 54, 967-978 (2012).

37. Shi, J., Cui, M., Yang, L., Kim, Y.-J. \& Zhang, D. Genetic and Biochemical Mechanisms of Pollen. Trends Plant. Sci. 20, 741-753 (2015).

38. $\mathrm{Ma}$, X. et al. A robust CRISPR/Cas9 system for convenient, high-efficiency multiplex genome editing in monocot and dicot plants. Mol. Plant 8, 1274-1284 (2015)

39. Maquat, L. E. Nonsense-mediated mRNA decay: splicing, translation and mRNP dynamics. Nat. Rev. Mol. Cell Biol. 5, 89-99 (2004).

40. Rieseberg, L. H. \& Blackman, B. K. Speciation genes in plants. Ann. Bot 106, 439-455 (2010)

41. Chandler, V. \& Stam, M. Chromatin conversations: Mechanisms and implications of paramutation. Nat. Rev. Genet. 5, 532-544 (2004).

42. Lawson, H. A., Cheverud, J. M. \& Wolf, J. B. Genomic imprinting and parentof-origin effects on complex traits. Nat. Rev. Genet. 14, 609-617 (2013).

43. Reinius, B. \& Sandberg, R. Random monoallelic expression of autosomal genes: stochastic transcription and allele-level regulation. Nat. Rev. Genet. 16, 653-664 (2015).

44. Chess, A. Random and non-random monoallelic expression. Neuropsychopharmacology 38, 55-61 (2013).

45. Xie, Y. et al. Suppression or knockout of $S a F / S a M$ overcomes the $S a$-mediated hybrid male sterility in rice. J. Integr. Plant Biol. 59, 669-679 (2017).

46. Xie, W. et al. Breeding signatures of rice improvement revealed by a genomic variation map from a large germplasm collection. Proc. Natl Acad. Sci. USA 112, E5411-E5419 (2015).

47. Wang, X. et al. Global genomic diversity of Oryza sativa varieties revealed by comparative physical mapping. Genetics 196, 937-949 (2014).
48. Liu, Y.-G. \& Whittier, R. F. Rapid preparation of megabase plant DNA from nuclei in agarose plugs and microbeads. Nucleic Acids Res. 22, 2168-2169 (1994)

49. Luo, D. et al. A detrimental mitochondrial-nuclear interaction causes cytoplasmic male sterility in rice. Nat. Genet. 45, 573-577 (2013).

50. Sato, Y. et al. RiceXPro version 3.0: Expanding the informatics resource for rice transcriptome. Nucleic Acids Res. 41, D1206-D1213 (2013).

51. Li, Y. D. et al. A cost-effective high-resolution melting approach using the EvaGreen dye for DNA polymorphism detection and genotyping in plants. $J$. Integr. Plant Biol. 52, 1036-1042 (2010).

52. Xie, X. et al. CRISPR-GE: A convenient software toolkit for CRISPR-based genome editing. Mol. Plant. 10, 1246-1249 (2017).

53. Liu, W. et al. DSDecode: a web-based tool for decoding of sequencing chromatograms for genotyping of targeted mutations. Mol. Plant 8, 1431-1433 (2015).

\section{Acknowledgements}

We thank G. Zhang, C. Zhuang and X.D. Liu (South China Agricultural University, SCAU) for providing T65, E5, and some other rice materials. We also thank $\mathrm{H}$. Wang (SCAU), D. Charlesworth (University of Edinburgh), K. Tsunewaki (Kyoto University) H. Ma (Fudan University), Q. Zhang and Y. Ouyang (Huazhong Agricultural University) for comments on the manuscript. This work was supported by grants from the Ministry of Science and Technology of China (2013CBA01401, 2013CB126904 and

2012AA10A303), the National Nature Science Foundation of China (31401449), and the Nature Science Foundation of Guangdong Province, China (S2012040007477).

\section{Author contributions}

R.S. performed most of the experiments; L.W., X.L., J.W., W.J., X.Z., X.X., Q.Z., H.T., Q.L., and L.C. performed some of the experiments. R.S. and Y.-G.L. analyzed the data. Y.-G.L. conceived and supervised the project, and Y.-G.L. and R.S. wrote the manuscript.

\section{Additional information}

Supplementary Information accompanies this paper at doi:10.1038/s41467-017-01400-y

Competing interests: The authors declare no competing financial interests.

Reprints and permission information is available online at http://npg.nature.com/ reprintsandpermissions/

Publisher's note: Springer Nature remains neutral with regard to jurisdictional claims in published maps and institutional affiliations.

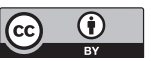

Open Access This article is licensed under a Creative Commons Attribution 4.0 International License, which permits use, sharing, adaptation, distribution and reproduction in any medium or format, as long as you give appropriate credit to the original author(s) and the source, provide a link to the Creative Commons license, and indicate if changes were made. The images or other third party material in this article are included in the article's Creative Commons license, unless indicated otherwise in a credit line to the material. If material is not included in the article's Creative Commons license and your intended use is not permitted by statutory regulation or exceeds the permitted use, you will need to obtain permission directly from the copyright holder. To view a copy of this license, visit http://creativecommons.org/ licenses/by/4.0/

(c) The Author(s) 2017 